\title{
Conservativity and time-flow invertibility of boundary control systems
}

Seville, 14 December 2005

Jarmo.Malinen@math.tkk.fi

Helsinki University of Technology 


\section{Overview}

In this talk, we

(i) explain the connection between boundary control systems (as defined below) and operator/system nodes; 


\section{Overview}

In this talk, we

(i) explain the connection between boundary control systems (as defined below) and operator/system nodes;

(ii) give sufficient and necessary conditions for such a boundary control system to define a (scattering) conservative system node (notion that has been defined in earlier literature); and 


\section{Overview}

In this talk, we

(i) explain the connection between boundary control systems (as defined below) and operator/system nodes;

(ii) give sufficient and necessary conditions for such a boundary control system to define a (scattering) conservative system node (notion that has been defined in earlier literature); and

(iii) present a PDE example involving the wave equation in $\mathbb{R}^{n}$ for $n \geq 2$. 


\section{Boundary nodes (1)}

Boundary control systems are described by the following equations

$$
\begin{cases}\dot{z}(t)=L z(t) & \text { (state dynamics) } \\ G z(t)=u(t) & \text { (input) } \\ y(t)=K z(t) & \text { (output) }\end{cases}
$$

for $t \geq 0$ where the operators

$$
L \in \mathcal{L}(\mathcal{Z} ; \mathcal{X}), \quad G \in \mathcal{L}(\mathcal{Z} ; \mathcal{U}) \quad \text { and } \quad K \in \mathcal{L}(\mathcal{Z} ; \mathcal{Y})
$$

and the Hilbert spaces $\mathcal{U}, \mathcal{X}, \mathcal{Y}$, and $\mathcal{Z}$ satisfy... 


\section{Boundary nodes (2)}

(i) $\mathcal{Z} \subset \mathcal{X}$ with a dense, continuous inclusion; 


\section{Boundary nodes (2)}

(i) $\mathcal{Z} \subset \mathcal{X}$ with a dense, continuous inclusion;

(ii) $\mathcal{U}=\operatorname{Ran} G$, and $\operatorname{Ker} G$ is dense in $\mathcal{X}$; 


\section{Boundary nodes (2)}

(i) $\mathcal{Z} \subset \mathcal{X}$ with a dense, continuous inclusion;

(ii) $\mathcal{U}=\operatorname{Ran} G$, and $\operatorname{Ker} G$ is dense in $\mathcal{X}$;

(iii) $(\alpha-L) \operatorname{Ker} G=\mathcal{X}$, and $\operatorname{Ker}(\alpha-L) \cap \operatorname{Ker} G=\{0\}$ for some $\alpha \in \overline{\mathbb{C}_{+}}$. 


\section{Boundary nodes (2)}

(i) $\mathcal{Z} \subset \mathcal{X}$ with a dense, continuous inclusion;

(ii) $\mathcal{U}=\operatorname{Ran} G$, and $\operatorname{Ker} G$ is dense in $\mathcal{X}$;

(iii) $(\alpha-L) \operatorname{Ker} G=\mathcal{X}$, and $\operatorname{Ker}(\alpha-L) \cap \operatorname{Ker} G=\{0\}$ for some $\alpha \in \overline{\mathbb{C}_{+}}$.

The triple $\Xi=(G, L, K)$ is called a boundary node. 


\section{Boundary nodes (2)}

(i) $\mathcal{Z} \subset \mathcal{X}$ with a dense, continuous inclusion;

(ii) $\mathcal{U}=\operatorname{Ran} G$, and $\operatorname{Ker} G$ is dense in $\mathcal{X}$;

(iii) $(\alpha-L) \operatorname{Ker} G=\mathcal{X}$, and $\operatorname{Ker}(\alpha-L) \cap \operatorname{Ker} G=\{0\}$ for some $\alpha \in \overline{\mathbb{C}_{+}}$.

The triple $\Xi=(G, L, K)$ is called a boundary node.

If $L \mid \operatorname{Ker} G$ generates a $C_{0}$-semigroup, we say that $\Xi$ is internally well-posed. 


\section{Boundary nodes (2)}

(i) $\mathcal{Z} \subset \mathcal{X}$ with a dense, continuous inclusion;

(ii) $\mathcal{U}=\operatorname{Ran} G$, and $\operatorname{Ker} G$ is dense in $\mathcal{X}$;

(iii) $(\alpha-L) \operatorname{Ker} G=\mathcal{X}$, and $\operatorname{Ker}(\alpha-L) \cap \operatorname{Ker} G=\{0\}$ for some $\alpha \in \overline{\mathbb{C}_{+}}$.

The triple $\Xi=(G, L, K)$ is called a boundary node.

If $L \mid \operatorname{Ker} G$ generates a $C_{0}$-semigroup, we say that $\Xi$ is internally well-posed.

There are many (essentially) equivalent definitions. 


\section{Connection to system nodes}

Internally well-posed boundary nodes $\Xi=(G, L, K)$ are in one-to-one correspondence with system nodes

$$
S=\left[\begin{array}{l}
A \& B \\
C \& D
\end{array}\right] \quad \text { on spaces }(\mathcal{U}, \mathcal{X}, \mathcal{Y})
$$

whose input operator $B$ is injective and strictly unbounded:

$$
\text { Ker } G=\{0\} \quad \text { and } \quad B \mathcal{U} \cap \mathcal{X}=\{0\} \text {. }
$$




\section{Connection to system nodes}

Internally well-posed boundary nodes $\Xi=(G, L, K)$ are in one-to-one correspondence with system nodes

$$
S=\left[\begin{array}{l}
A \& B \\
C \& D
\end{array}\right] \quad \text { on spaces }(\mathcal{U}, \mathcal{X}, \mathcal{Y})
$$

whose input operator $B$ is injective and strictly unbounded:

$$
\text { Ker } G=\{0\} \quad \text { and } \quad B \mathcal{U} \cap \mathcal{X}=\{0\} \text {. }
$$

Such system nodes $S$ are said to be of boundary control type. 


\section{Given $\Xi=(G, L, K) \ldots$}

...you get the corresponding $S=\left[\begin{array}{c}A \& B \\ C \& D\end{array}\right]$ from equations $A \& B\left[\begin{array}{l}x \\ u\end{array}\right]:=A_{-1} x+B u$ and $C \& D\left[\begin{array}{l}x \\ u\end{array}\right]:=K x$ where

(i) $\operatorname{dom}(A):=\operatorname{Ker} G$ and $A:=L \mid \operatorname{dom}(A)$;

(ii) $\mathcal{X}_{-1}:=\operatorname{dom}\left(A^{*}\right)^{d}$ using $\mathcal{X}$ as the pivot space, and the usual Yoshida extension $A_{-1}: \mathcal{X} \rightarrow \mathcal{X}_{-1}$;

(iii) $B G z:=L z-A_{-1} z \quad$ for all $z \in \mathcal{Z}$;

(iv) and $\operatorname{dom}(S):=\left[\begin{array}{l}I \\ G\end{array}\right] \mathcal{Z}$. 


\section{Given $\Xi=(G, L, K) \ldots$}

...you get the corresponding $S=\left[\begin{array}{c}A \& B \\ C \& D\end{array}\right]$ from equations $A \& B\left[\begin{array}{l}x \\ u\end{array}\right]:=A_{-1} x+B u$ and $C \& D\left[\begin{array}{l}x \\ u\end{array}\right]:=K x$ where

(i) $\operatorname{dom}(A):=\operatorname{Ker} G$ and $A:=L \mid \operatorname{dom}(A)$;

(ii) $\mathcal{X}_{-1}:=\operatorname{dom}\left(A^{*}\right)^{d}$ using $\mathcal{X}$ as the pivot space, and the usual Yoshida extension $A_{-1}: \mathcal{X} \rightarrow \mathcal{X}_{-1}$;

(iii) $B G z:=L z-A_{-1} z \quad$ for all $z \in \mathcal{Z}$;

(iv) and $\operatorname{dom}(S):=\left[\begin{array}{l}I \\ G\end{array}\right] \mathcal{Z}$.

(Don't worry. You need not memorize them right now.) 


\section{The Cauchy problem (1)}

Assume: Boundary node $\Xi=(G, L, K)$ is internally well-posed; $u \in C^{2}([0, \infty) ; \mathcal{U})$ and $z_{0} \in \mathcal{Z}$ satisfy the compatibility condition $G z_{0}=u(0)$. 


\section{The Cauchy problem (1)}

Assume: Boundary node $\Xi=(G, L, K)$ is internally well-posed; $u \in C^{2}([0, \infty) ; \mathcal{U})$ and $z_{0} \in \mathcal{Z}$ satisfy the compatibility condition $G z_{0}=u(0)$.

Then: the equations for $t \geq 0$

$$
\dot{z}(t)=L z(t), \quad G z(t)=u(t), \quad y(t)=K z(t),
$$

have a unique solution $z(\cdot) \in C([0, \infty) ; \mathcal{Z}) \cap$ $C^{1}([0, \infty) ; \mathcal{X})$, such that $z(0)=z_{0}$ and $y(\cdot) \in$ $C([0, \infty) ; \mathcal{Y})$ 


\section{The Cauchy problem (2)}

And also: the same functions $u(\cdot), z(\cdot)$ and $y(\cdot)$ satisfy

$$
\dot{z}(t)=A_{-1} z(t)+B u(t), \quad y(t)=C \& D\left[\begin{array}{c}
z(t) \\
u(t)
\end{array}\right],
$$

for $t \geq 0$. Here the system node

$$
S=\left[\begin{array}{l}
A \& B \\
C \& D
\end{array}\right]
$$

corresponds to the boundary node $\Xi=(G, L, K)$ in the way described above. 


\section{Conservativity of system nodes}

The system node $S=\left[\begin{array}{c}A \& B \\ C \& D\end{array}\right]$ is (scattering) energy preserving if for any $u(\cdot) \in C^{2}\left(\mathbb{R}_{+} ; \mathcal{U}\right)$ and any (compatible) initial state $z(0)=z_{0}$, the solution of

$$
\dot{z}(t)=A_{-1} z(t)+B u(t), \quad y(t)=C \& D\left[\begin{array}{c}
z(t) \\
u(t)
\end{array}\right]
$$

satisfies the energy balance equation

$$
\frac{d}{d t}\|x(t)\|_{\mathcal{X}}^{2}=\|u(t)\|_{\mathcal{U}}^{2}-\|y(t)\|_{\mathcal{Y}}^{2}
$$




\section{Conservativity of system nodes}

The system node $S=\left[\begin{array}{c}A \& B \\ C \& D\end{array}\right]$ is (scattering) energy preserving if for any $u(\cdot) \in C^{2}\left(\mathbb{R}_{+} ; \mathcal{U}\right)$ and any (compatible) initial state $z(0)=z_{0}$, the solution of

$$
\dot{z}(t)=A_{-1} z(t)+B u(t), \quad y(t)=C \& D\left[\begin{array}{c}
z(t) \\
u(t)
\end{array}\right]
$$

satisfies the energy balance equation

$$
\frac{d}{d t}\|x(t)\|_{\mathcal{X}}^{2}=\|u(t)\|_{\mathcal{U}}^{2}-\|y(t)\|_{\mathcal{Y}}^{2}
$$

$S$ is conservative, if both $S$ and the dual node $S^{d}$ are energy preserving. 


\section{Why is this definition "the right one"?}

This definition of conservativity can be defended from several directions:

(i) It is a generalization from the finite dimensions; 


\section{Why is this definition "the right one"?}

This definition of conservativity can be defended from several directions:

(i) It is a generalization from the finite dimensions;

(ii) By the Cayley transform, it is equivalent to the usual discrete time definition; 


\section{Why is this definition "the right one"?}

This definition of conservativity can be defended from several directions:

(i) It is a generalization from the finite dimensions;

(ii) By the Cayley transform, it is equivalent to the usual discrete time definition;

(iii) It is equivalent to the old definition of the operator colligation by Brodskiĭ, Livšic, Sz.-Nagy \&al. in the theory of Hilbert space contractions; 


\section{Why is this definition... (cont'd)}

(iv) System theoretically, it is a very "happy class" e.g. a strong form of the state space isomorphism theorem holds. 


\section{Why is this definition... (cont'd)}

(iv) System theoretically, it is a very "happy class" e.g. a strong form of the state space isomorphism theorem holds.

(v) As this work shows, it relates in the right way to the time-flow invertibility - an important property of hyperbolic linear PDEs. 


\section{Why is this definition... (cont'd)}

(iv) System theoretically, it is a very "happy class" e.g. a strong form of the state space isomorphism theorem holds.

(v) As this work shows, it relates in the right way to the time-flow invertibility - an important property of hyperbolic linear PDEs.

(vi) As our newer work shows, it relates (after a translation to "impedance setting") in the right way to the abstract boundary spaces, used for extensions of symmetric operators in Russian literature. 


\section{How about conservative boundary nodes?}

Question: How to characterize those conservative boundary nodes $\Xi=(G, L, K)$ that correspond to conservative system nodes as described above? 


\section{How about conservative boundary nodes?}

Question: How to characterize those conservative boundary nodes $\Xi=(G, L, K)$ that correspond to conservative system nodes as described above?

Practical problems:

(i) The translation of the data $\Xi=(G, L, K)$ to an operator node $S$ is cumbersome (especially if $\Xi$ comprises partial differential operators!) 


\section{How about conservative boundary nodes?}

Question: How to characterize those conservative boundary nodes $\Xi=(G, L, K)$ that correspond to conservative system nodes as described above?

Practical problems:

(i) The translation of the data $\Xi=(G, L, K)$ to an operator node $S$ is cumbersome (especially if $\Xi$ comprises partial differential operators!)

(ii) The dual system $S^{d}$ need not be of boundary control type, even if $S$ is; $\Rightarrow$ the direct, pure translation of the definition to boundary nodes is impossible! 


\section{Characterization of conservative}

$$
\Xi=(G, L, K)
$$

The triple $\Xi=(G, L, K)$ is a doubly boundary node, if both $\Xi$ and $\Xi^{\leftarrow}:=(K,-L, G)$ are boundary nodes. 


\section{Characterization of conservative}

$$
\Xi=(G, L, K)
$$

The triple $\Xi=(G, L, K)$ is a doubly boundary node, if both $\Xi$ and $\Xi^{\leftarrow}:=(K,-L, G)$ are boundary nodes.

Theorem 1: Let $\Xi=(G, L, K)$ be a doubly boundary node, and by $S=\left[\begin{array}{c}A \& B \\ C \& D\end{array}\right]$ denote the associated operator node. Then $S$ is conservative if and only if

(i) $2 \Re\langle x, L x\rangle_{\mathcal{X}}=-\|K x\|_{\mathcal{Y}}^{2}$ for all $x \in \operatorname{Ker} G$,

(ii) $\langle z, L x\rangle_{\mathcal{X}}+\langle L z, x\rangle_{\mathcal{X}}=\langle G z, G x\rangle_{\mathcal{U}}$ for all $z \in \mathcal{Z}$ and $x \in \operatorname{Ker} K$. 


\section{“Childrens version"}

There is another variant whose formulation is more beautiful. 


\section{“Childrens version"}

There is another variant whose formulation is more beautiful.

Theorem 2: Let $\Xi=(G, L, K)$ be a doubly boundary node, and by $S=\left[\begin{array}{c}A \& B \\ C \& D\end{array}\right]$ denote the associated operator node.

Then $S$ is conservative if and only if the GreenLagrange identity

$$
2 \Re\left\langle z_{0}, L z_{0}\right\rangle_{\mathcal{X}}=\left\|G z_{0}\right\|_{\mathcal{U}}^{2}-\left\|K z_{0}\right\|_{\mathcal{Y}}^{2}
$$

holds for all $z_{0} \in \mathcal{Z}$. 


\section{References to the proofs}

The proof of Theorem 1 . is based on the characterization of conservative system nodes among timeflow invertible system nodes [Malinen; (2004, 2005)], in combination with the main theorem of [Malinen, Staffans, Weiss; $(2003,2005)]$ on "tory" systems. 


\section{References to the proofs}

The proof of Theorem 1 . is based on the characterization of conservative system nodes among timeflow invertible system nodes [Malinen; (2004, 2005)], in combination with the main theorem of [Malinen, Staffans, Weiss; $(2003,2005)]$ on "tory" systems.

The proof of the slightly weaker Theorem 2. can be carried out alternatively by a direct argument, see [Malinen, Staffans; (2005)]. 


\section{References to the proofs}

The proof of Theorem 1 . is based on the characterization of conservative system nodes among timeflow invertible system nodes [Malinen; (2004, 2005)], in combination with the main theorem of [Malinen, Staffans, Weiss; $(2003,2005)]$ on "tory" systems.

The proof of the slightly weaker Theorem 2. can be carried out alternatively by a direct argument, see [Malinen, Staffans; (2005)].

Theorem 1. can be also concluded from Theorem 2. by using the main theorem of [Malinen, Staffans, Weiss; $(2003,2005)]$. 


\section{The scattering conservative wave equation (1)}

Suppose $\Omega \subset \mathbb{R}^{n}, n \geq 2$, is an open bounded set with $C^{2}$-boundary $\partial \Omega$.

We assume that $\partial \Omega$ is the union of two sets $\Gamma_{0}$ and $\Gamma_{1}$ with $\overline{\Gamma_{0}} \cap \overline{\Gamma_{1}}=\emptyset$. 


\section{The scattering conservative wave equation (1)}

Suppose $\Omega \subset \mathbb{R}^{n}, n \geq 2$, is an open bounded set with $C^{2}$-boundary $\partial \Omega$.

We assume that $\partial \Omega$ is the union of two sets $\Gamma_{0}$ and $\Gamma_{1}$ with $\overline{\Gamma_{0}} \cap \overline{\Gamma_{1}}=\emptyset$.

In the same PDE example, the sets $\Gamma_{1}$ and $\Gamma_{0}$ are allowed to have zero distance in [Weiss, Tucsnak; (2003)]. This is possible because stronger "background results" from [Rodrigues-Bernal, Zuazua; (1995)] are used there. 


\section{The scattering conservative wave equation (2)}

We are interested in the system node $S$ that (hopefully) is described by the exterior problem

$$
\left\{\begin{array}{l}
z_{t t}(t, \xi)=\Delta z(t, \xi) \quad \text { for } \xi \in \Omega \text { and } t \geq 0, \\
-z_{t}(t, \xi)-\frac{\partial z}{\partial \nu}(t, \xi)=\sqrt{2} u(t, \xi) \quad \text { for } \xi \in \Gamma_{1} \text { and } t \geq 0, \\
\sqrt{2} y(t, \xi)=-z_{t}(t, \xi)+\frac{\partial z}{\partial \nu}(t, \xi) \quad \text { for } \xi \in \Gamma_{1} \text { and } t \geq 0, \\
z(t, \xi)=0 \quad \text { for } \xi \in \Gamma_{0} \text { and } t \geq 0, \text { and } \\
z(0, \xi)=z_{0}(\xi), \quad z_{t}(0, \xi)=w_{0}(\xi) \quad \text { for } \xi \in \Omega .
\end{array}\right.
$$

Note that $\Gamma_{0}$ is the reflecting part of $\partial \Omega$. 


\section{The scattering conservative wave equation (3)}

We discover the boundary node $\Xi=(G, L, K)$ by

$$
z_{t t}=\Delta z \quad \hat{=} \frac{d}{d t}\left[\begin{array}{c}
z \\
w
\end{array}\right]=\left[\begin{array}{cc}
0 & -1 \\
-\Delta & 0
\end{array}\right]\left[\begin{array}{c}
z \\
w
\end{array}\right]
$$




\section{The scattering conservative wave equation (3)}

We discover the boundary node $\Xi=(G, L, K)$ by

$$
z_{t t}=\Delta z \quad \hat{=} \frac{d}{d t}\left[\begin{array}{c}
z \\
w
\end{array}\right]=\left[\begin{array}{cc}
0 & -1 \\
-\Delta & 0
\end{array}\right]\left[\begin{array}{c}
z \\
w
\end{array}\right]
$$

The spaces $\mathcal{Z}, \mathcal{X}$ and and operator $L$ are defined by

$$
\begin{gathered}
L:=\left[\begin{array}{cc}
0 & -1 \\
-\Delta & 0
\end{array}\right]: \mathcal{Z} \rightarrow \mathcal{X} \text { with } \\
\mathcal{Z}:=\mathcal{Z}_{0} \times H_{\Gamma_{0}}^{1}(\Omega) \text { and } \mathcal{X}:=H_{\Gamma_{0}}^{1}(\Omega) \times L^{2}(\Omega)
\end{gathered}
$$

where $\mathcal{Z}_{0}:=\left\{z \in H_{\Gamma_{0}}^{1}(\Omega) \cap H^{3 / 2}(\Omega): \Delta z \in L^{2}(\Omega)\right\}$. 


\section{The scattering conservative wave equation (4)}

The norm of $\mathcal{Z}_{0}$ is given by

$$
\left\|z_{0}\right\|_{\mathcal{Z}_{0}}^{2}:=\left\|z_{0}\right\|_{H^{1}(\Omega)}^{2}+\left\|z_{0}\right\|_{H^{3 / 2}(\Omega)}^{2}+\left\|\Delta z_{0}\right\|_{L^{2}(\Omega)}^{2} .
$$




\section{The scattering conservative wave equation (4)}

The norm of $\mathcal{Z}_{0}$ is given by

$$
\left\|z_{0}\right\|_{\mathcal{Z}_{0}}^{2}:=\left\|z_{0}\right\|_{H^{1}(\Omega)}^{2}+\left\|z_{0}\right\|_{H^{3 / 2}(\Omega)}^{2}+\left\|\Delta z_{0}\right\|_{L^{2}(\Omega)}^{2} .
$$

For the state space $\mathcal{X}$, we use the energy norm

$$
\left\|\left[\begin{array}{c}
z_{0} \\
w_{0}
\end{array}\right]\right\|_{\mathcal{X}}^{2}:=\left\|\mid \nabla z_{0}\right\|_{L^{2}(\Omega)}^{2}+\left\|w_{0}\right\|_{L^{2}(\Omega)}^{2} .
$$




\section{The scattering conservative wave equation (5)}

Define the input and output spaces by setting $\mathcal{U}=\mathcal{Y}:=L^{2}\left(\Gamma_{1}\right)$, together with

$$
\begin{aligned}
G\left[\begin{array}{c}
z_{0} \\
w_{0}
\end{array}\right] & :=\frac{1}{\sqrt{2}}\left(-\frac{\partial z_{0}}{\partial \nu}\left|\Gamma_{1}+w_{0}\right| \Gamma_{1}\right) \text { and } \\
K\left[\begin{array}{c}
z_{0} \\
w_{0}
\end{array}\right] & :=\frac{1}{\sqrt{2}}\left(\frac{\partial z_{0}}{\partial \nu}\left|\Gamma_{1}+w_{0}\right| \Gamma_{1}\right) .
\end{aligned}
$$




\section{The scattering conservative wave equation (5)}

Define the input and output spaces by setting $\mathcal{U}=\mathcal{Y}:=L^{2}\left(\Gamma_{1}\right)$, together with

$$
\begin{aligned}
G\left[\begin{array}{c}
z_{0} \\
w_{0}
\end{array}\right] & :=\frac{1}{\sqrt{2}}\left(-\frac{\partial z_{0}}{\partial \nu}\left|\Gamma_{1}+w_{0}\right| \Gamma_{1}\right) \text { and } \\
K\left[\begin{array}{c}
z_{0} \\
w_{0}
\end{array}\right] & :=\frac{1}{\sqrt{2}}\left(\frac{\partial z_{0}}{\partial \nu}\left|\Gamma_{1}+w_{0}\right| \Gamma_{1}\right) .
\end{aligned}
$$

We have now the triple of operators $\Xi=(G, L, K)$, together with the Hilbert spaces $\mathcal{U}, \mathcal{X}, \mathcal{Y}$ and $\mathcal{Z}$. 


\section{The scattering conservative wave equation (6)}

Proposition 3: The triple of operators $\Xi=(G, L, K)$ defined above is a doubly boundary node on spaces $\mathcal{U}$, $\mathcal{X}, \mathcal{Y}$ and $\mathcal{Z}$. 


\section{The scattering conservative wave equation (6)}

Proposition 3: The triple of operators $\Xi=(G, L, K)$ defined above is a doubly boundary node on spaces $\mathcal{U}$, $\mathcal{X}, \mathcal{Y}$ and $\mathcal{Z}$.

The proof requires well-known properties of the Sobolev spaces (like the Poincaré inequality), standard results on Dirichlet and Neumann traces, and elliptic regularity theory. 


\section{The scattering conservative wave equation (6)}

Proposition 3: The triple of operators $\Xi=(G, L, K)$ defined above is a doubly boundary node on spaces $\mathcal{U}$, $\mathcal{X}, \mathcal{Y}$ and $\mathcal{Z}$.

The proof requires well-known properties of the Sobolev spaces (like the Poincaré inequality), standard results on Dirichlet and Neumann traces, and elliptic regularity theory.

We now know that there exists a unique system node $S=\left[\begin{array}{c}A \& B \\ C \& D\end{array}\right]$ associated to $\Xi$. 


\section{The scattering conservative wave equation (7)}

Proposition 4: Let the boundary node $\Xi=(G, L, K)$ be defined as above. Use the energy norm

$$
\left\|\left[\begin{array}{c}
z_{0} \\
w_{0}
\end{array}\right]\right\|_{\mathcal{X}}^{2}:=\left\|\left|\nabla z_{0}\right|\right\|_{L^{2}(\Omega)}^{2}+\left\|w_{0}\right\|_{L^{2}(\Omega)}^{2} .
$$

for the state space $\mathcal{X}$. Then the system node $S$ associated to $\Xi$ is conservative. 


\section{The scattering conservative wave equation (7)}

Proposition 4: Let the boundary node $\Xi=(G, L, K)$ be defined as above. Use the energy norm

$$
\left\|\left[\begin{array}{c}
z_{0} \\
w_{0}
\end{array}\right]\right\|_{\mathcal{X}}^{2}:=\left\|\left|\nabla z_{0}\right|\right\|_{L^{2}(\Omega)}^{2}+\left\|w_{0}\right\|_{L^{2}(\Omega)}^{2} .
$$

for the state space $\mathcal{X}$. Then the system node $S$ associated to $\Xi$ is conservative.

Indeed, the conditions of Theorem 2. can be checked by using a generalized Greens formula. 


\section{The scattering conservative wave equation (7)}

Proposition 4: Let the boundary node $\Xi=(G, L, K)$ be defined as above. Use the energy norm

$$
\left\|\left[\begin{array}{c}
z_{0} \\
w_{0}
\end{array}\right]\right\|_{\mathcal{X}}^{2}:=\left\|\left|\nabla z_{0}\right|\right\|_{L^{2}(\Omega)}^{2}+\left\|w_{0}\right\|_{L^{2}(\Omega)}^{2} .
$$

for the state space $\mathcal{X}$. Then the system node $S$ associated to $\Xi$ is conservative.

Indeed, the conditions of Theorem 2. can be checked by using a generalized Greens formula.

A numerical example will be given later by $\mathrm{V}$. Havu. 


\section{References}

Boundary control, operator nodes in general:

H. O. Fattorini. Boundary control systems. SIAM J. Control, 6(3), 1968.

M. S. Brodskir. Triangular and Jordan representations of linear operators, volume 32. AMS, 1971.

D. Salamon. Infinite dimensional linear systems with unbounded control and observation: a functional analytic approach. Trans. AMS, 300:383-431, 1987.

D. Salamon. Realization theory in Hilbert spaces. Math. Systems Theory, 21:147-164, 1989. 
O. J. Staffans. Well-Posed Linear Systems. Cambridge University Press, 2004.

B. Sz.-Nagy and C. Foias. Harmonic Analysis of Operators on Hilbert space. North-Holland Publishing Company, 1970. 


\section{Related to conservative systems:}

D. Z. Arov and M. A. Nudelman. Passive linear stationary dynamical scattering systems with continuous time. Int. Eq. Oper. Th., 24:1-45, 1996.

M. S. Livšic and A. A. Yantsevich. Operator colligations in Hilbert space. John Wiley \& sons, Inc., 1977.

V. Havu and J. Malinen. Laplace and Cayley transforms - an approximation point of view, Proc. of the CDC-ECC'05, 2005.

J. Malinen. Conservativity of time-flow invertible and boundary control systems. Helsinki Univ. of Tech. Inst. of Math. Research Reports A479, 2004. 
J. Malinen and O. J. Staffans. Conservative boundary control systems. Submitted, 2005.

J. Malinen, O. Staffans, and G. Weiss. When is a linear system conservative? Quart. Appl. Math., 2005.

G. Weiss and M. Tucsnak. How to get a conservative well-posed linear system out of thin air. I. Wellposedness and energy balance. ESAIM Control Optim. Calc. Var., 9:247-274, 2003. 


\section{Papers related to the wave equation example:}

J. Lagnese. Decay of solutions of wave equations in a bounded region with boundary dissipation. J. Diff. Eq., 50:163-182, 1983.

A. Rodrígues-Bernal and E. Zuazua. Parabolic singular limit of a wave equation with localized boundary damping. Discrete Contin. Dynam. Systems, 1(3):303-346, 1995.

R. Triggiani. Wave equation on a bounded domain with boundary dissipation: An operator approach. J. Math. Anal. Appl., 137:438-461, 1989. 


\title{
That's all of it, folks!
}

\author{
Have a nice day.
}

